\title{
Autorenverzeichnis 1990
}

Achterrath,W.,190 Adam, D., 38 Ade,N.,359 Adler,A.,272 Adler,M.,313 Albrecht, U.,313

Alhusen,R.,210 Allmayer, H.,310 Ammon, A.,,210, 272 Ammon, J., 144 Andreopoulos, D., 144 Anger, B., 109 Arnold,H., 253 Atzinger, A.,260 Aulbert,E.,102

Bätz,W.,21 Barnickel, I., 364 Bartels,H.,458 Bartl,R.,458 Bartsch,H. H.,137,210 Bastert,G.,394 Becker, H., 381 Berdel,W. E.,180,245,

369, 437, 444 Berger, M. R.,17 Berrgmann, L.,137,416 Beuth,J.,124 Beyer, J.-H., 175,190, 272 Birnbach,A.,280 Blumenstein, M.,289 Bodemann, H. , 253 Boerner, D., 322 Bois, A. du, 364 Braasch,D.,381 Brack-Werner, R., 405 Braumann, D.,465 Braun,H. J.,458 Broen, I.-M. von, 43, 458 Bruijn, E. A. de, 203 Brunner, T.,429 Bruntsch,U.,28 Bülow, M. von, 21 Bülzingslöwen, F. von, 253 Burk,K.,346 Busse,E.,12, 301

Calavrezos, A., 132 Cammerer, U., 43 Carbonell, F.,109 Coldewey,R.,43,458 Cour, V.,7 CroneMuenzebrock, L. S.,354

Danhauser-Riedl, 437 Deicher, PL,43,458 Dietel,M.,448 van Dongen, J. J. M., 166

Dopfer, R.,429 Drings, P., 28,141,180, 186

Ebener, C.,381 Edler, L.,17,50,63,90,

$175,180,186,190,194$,

322, 444 Ehninger, G.,429 Ehrhart, H.,17 Eibl,H.,295

Eiermann, W., 394

Eiffert,H.,210

Erfle,V.,405

Ertl,A.,369

Eschenbach, I., 28

Essers, U., 175

Exeriede, G.,280

Fiebig,H. H.,175,186 Fink, M., 17 Fink, U., 369 Fischer, H., 305 Fischer,J.T., 458 Flasshove, M.,33 Flechtner,H.,50,194 Fleer,E. A.M., 295 Franks, C. R., 137 Freund,M.,280,424 Fritz, E., 46 Fritz, M., 50 Fritze,D.,190 Füger, K.,289

Ganser, A., 33,109 Garbrecht, M.,354 Gatzemeier, U., 180,

186, 285 Gheuens, E., 203 Gisslinger, H.,46 Goßmann,H. H.,28 Gramatzki, M.,458 Greifenberg, B., 175 Grill, H.-J., 21 Groh,E.,210 Gropp, C, 28 Grothey, A.,33 Grundmann, R., 124

Günther,I.U.,180 Günzl, C.,465 Gutt,C. N.,21

Hanauske, A. R.,424 Harjung, H., 194 Hartlapp, J.,24 Hastka,J.,317 Havemann,K.,28,157

Heckmayr, M.,285 Hehlmann, R.,405 Heidemann,E.,24, 338 Heilmann,H. P.,132

Heim,M.E.,50,63,317, 359, 444 Heimpel,H.,109 Hein,R.,43,458 Helmerking, M., 38 Henke,R.,458 Henkel,E.,375 Henne-Bruns, D., 354 Herrmann, R., 63, 322 Hiddemann,W.,38 Hinke, A., 186, 322 Hinkelbein,W.,253 Höffken,K.,33,237 Hoelzer, D.,33 Hoffmann, L., 43, 458 Hoffmann,W., 217 Hofmann, J., 166 Holdener, E.E.,117 Holle,R.,28 Holtkamp,W.,207 Hossfeld,D.K.,24,63, 
346, 354, 448

Illiger,H.J.,444 Ingersleben, G. von, 24 Issinger, O.-G., 305

Jaenicke, F., 313 Jonat,W.,394 Jost,L. M.,96

Kabelitz, K.,194 Kaplan, E., 322 Karstens, J.H., 144 Kau,B.,453 Kaufmann,M.,394

Keilholz,U.,137 Kim,D.-J.,295 Kiss, D., 388 Klee,M.,444 Kleeberg,U.R.,175 Klein, H.O., 63

Kleine,H.D.,280 Klocker,J.,310 Kluger,J.,124 Ko,H.L.,124 Köppler, H.,28 Konyar, H.,43

Kornhuber, B.,12,301 Kotzmann, H., 46 Kraft, A., 253 Krammer, B.,462 Kremer, B.,354

Kretschmar, A., 141 Kreuser, E.D.,43,458 Küchler, R.,132 Kuhn,H.,28 Kuhn,W.,268

Kunz,S.,24 Kuse, R.,132

Lagoda, P.,305 Langenbahn, H.,7 Langenbuch, T., 346 Leib-Mösch,C.,405 Lennert, K.,28

Lenz,H.J.,429 Leo, R., 458 Lewerenz, B., 465 Lindner, H., 260 Link, H., 38, 280 Lötzke,E.,28

Ludwig,Ch.U.,117 Ludwig, H.,46 Ludwig,W.-D.,166 Ludwig-Hagemann, R., 117

Maass,H.,394 Maier,W.D.,458 Mainzer, K.,465 Manegold, C.,194 Marschner,N.,210,272

Maschmeyer, G., 38 Meerpohl,H.G.,364 Meier,C.R., 458 Mentges,B.,21 Meyer, P., 38 Mitrou, P.S.,137 Mross, K.B.,190,346

Nagel, G.A.,207,272,

295,313 Neeser, E.,24 Neuhaus,K.L.,221

Noder,W.,17

Obrecht,J.P.,63,117,

333 Obrist,R., 117,388 Oehl, S.,28 Oertel, J.,458 Osmers, R.,268 Overkamp,F.,22

Pechan,R.,322 Peest,D.,43,458 Perker,M.,369 Peters, K.M., 124 Peukert, M.,322 Pfab,R.,28

Pfleiderer, A.,364 Pflügler,K.H.,28 Ranker, M., 458 Poliwoda, H.,280 Pollow, K.,21 Possinger, K.,24 Preusser,P.,63,190 Pulverer, G.,124

Quaas, L.,364 Queißer,W.,50,63,190, 194, 317,322

Rab,B.,310 Rath, U., 194 Rastetter, J.,437 Rath, W., 268 Rauschecker, H., 207 Reichel,L.,175

Reichold,M.,369 Reínhold, H.M.,458 Reis,H.E.,207 Rieche,K., 186,190,444 Rieder, H.,322

Rieger, M.,453 Riehm,H.,166 Robertson, J., 109 Röher,H.D.,381 Rosen, H.R., 128

Rümelin,B.,210 Runne,U.,137

Salewski,E.,175,180 Salmons, B., 405 Sass,H.-M.,85 Sauer,H.,289,453 Seeber, S.,217

Selbach,J., 43,194,458 Serve, H., 369 Siegmund,R.,444 Sinop, A., 17 Slee,P.H.Th.J.,203

Sokal,J.E.,109 Spoula,H.,128 Sutter-Melde,C.,117

Schäfer, E.,458 Scherübl,H.,198 Schlag,H.,429 Schlag,P.,194 Schmähl,D.,17 Schmeiser,T., 109

Schmidt, J., 405 Schmoll, H.J.,63 Schneider, G., 359 Schubert-Fritschle, G., 260

Schumacher, E., 458 Schumacher, K., 24, 458 Schumer, J., 310 Schuster, D., 359 Schwarz,

R.,448

Stahl,M.,180 Steffens,O.,102 Stein,H., 28 Steinhauer,E.U.,28 Steinhauser, G., 437

Steinke,B.,24, 63 Stennes, M.,458 Stenzinger, W.,458 Sterz, R.,322 Stierer,M.,128 Stirbu,J.,33

Strauss, P.G., 405

Teichmann, J.V., 166

Tessen,H.W.,186

Thiel,E.,166

Thiery,J.,207

Tidstrand,J.,310

Tirier,C.,458

Trux,F.,50,194

Türeci,O.,305 
Unger, C.,295 Unterburger, P., 17

Verhees,S.,50 Völksen,M.,268

Waldmann,R.,24 Wagner, H., 458 Walter, T.A., 354 Wander, H,-E., 175 Wannenmacher, M., 253 Weber, M., 210 Wegmann,W.,388 Weh,H.J.,43,63,354,

448,458 Weidmann,B.,217 Weidmann, E., 137 Wilhelm,C.,364 Wilke,H.,63 Willich,N.,260

Wingberg, D.,448 Wolf, M., 157 Wysk, J.,458

Zeller,W.,28

Zeymer, U.,221

Ziegler,R.,198

Zoedler,T.,381

Zornig, C.,448

Zügel,M.,448

Zwingers,T.,253

(Autoren von ABSTRACTS sind nicht im Jahresgesamtver-zeichnis aufgeführt) 